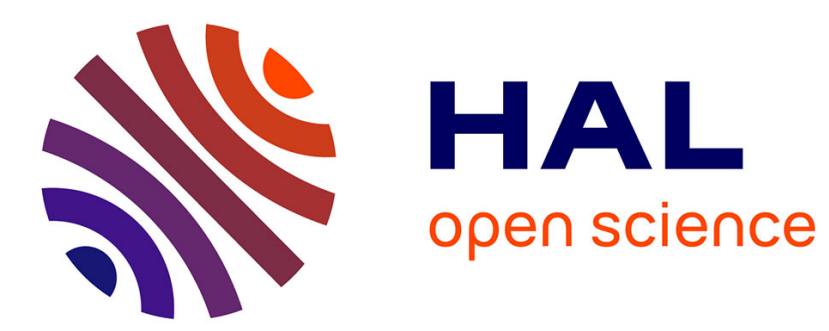

\title{
Positive Relations Between Vision and Posture in the Fixation Task Performed Upright.
}

\author{
Cédrick T. Bonnet
}

\section{To cite this version:}

Cédrick T. Bonnet. Positive Relations Between Vision and Posture in the Fixation Task Performed Upright.. Motor Control, 2019, 24 (1), pp.1-16. 10.1123/mc.2018-0094 . hal-02525772v2

HAL Id: hal-02525772

https://hal.univ-lille.fr/hal-02525772v2

Submitted on 3 Apr 2020 (v2), last revised 10 Aug 2021 (v3)

HAL is a multi-disciplinary open access archive for the deposit and dissemination of scientific research documents, whether they are published or not. The documents may come from teaching and research institutions in France or abroad, or from public or private research centers.
L'archive ouverte pluridisciplinaire HAL, est destinée au dépôt et à la diffusion de documents scientifiques de niveau recherche, publiés ou non, émanant des établissements d'enseignement et de recherche français ou étrangers, des laboratoires publics ou privés. 


\title{
Positive Relations Between Vision and Posture in the Stationary-Gaze ask Performed Upright
}

\author{
Cédrick T. Bonnet \\ University of Lille
}

In an upright stance, individuals sway in unpredictable ways. Their eyes also move in unpredictable ways in fixation tasks. The objective of this study was to analyze visual functions, postural control, and cognitive involvement in the stationary gaze A total of 14 healthy young adults performed a stationary-gaze task and a free viewing task (three trials per task, $45 \mathrm{~s}$ per trial). As expected, the results showed many $(n=32)$ significant positive Pearson correlation coefficients between the eye and center of pressure/body (head, neck, and lower back) movements in the stationary-gaze task an $I$ nonsignificant in free-viewing task. Only 3 of the 32 significant correlatic... $9.4 \%$ ) were significantly related to cognitive involvement (measured with a subjective questionnaire). As strengthened the validity of the synergistic mo f postural control.

Keywords: cognitive involvement, correlations, eye body-related movements, quiet stance, young adults

In the literature on postural control, many investigators have studied the movements of the body in one or several tasks and used the stationary-gaze task to provide baseline data to better understand their results (e.g., Kapteyn et al., 1983; Raymakers, Samson, \& Verhaar, 2005). In this stationary-gaze task, participants generally look at a stationary target in front of them, located at eye height. This task seems very easy and basic. However, it may not be so easy to maintain the eyes on a stationary target because individuals sway continuously in an irregular, nonlinua, and nonstationary way (Bonnet et al., 2010; Collins \& DeLuca, 1995; Riley \& Turvey, 2002). Moreover, microsaccades can alter the stationary gaze (OteroMillan, Troncoso, Macknik, Serrano-Pedraza, \& Martinez-Conde, 2008; Thaler Schütz, Goodale, \& Gegenfurtner, 2013), as they are erratic (Engbert \& Kliegl, 2004). Consequently, in a stationary-gaze task performed upright, the images on the retinas arr always changing (Aytekin \& Rucci, 2012).

In th ...erature reports, many investigators have studied the interaction between visual functions and postural control in an upright stance (e.g., Giveans

The author is with SCALab, CNRS UMR 9193, University of Lille, Lille, France. Bonnet (cedrick. 
Yoshida, Bardy, Riley, \& Stoffregen, 2011; Legrand et al., 2013; Rougier \& Garin, 2007; Schulmann, Godfrey, \& Fisher, 1987; Stoffregen, Bardy, Bonnet, Hove, \& Oullier, 2007; Thomas, Bampouras, Donova, \& Dewhurst, 2016). In these studies, the stationary-gaze task was systematically used as a basic or control task, but never as the task of interest. These studies generally analyzed how pursuit visual tasks (Schulmann et al., 1987; Thomas et al., 2016) and/or saccadic left-right tasks (Giveans et al., 2011; Rougier \& Garin, 2007) and/or precise detections (Legrand et al., 2013; Stoffregen et al., 2007) could influence postural control. In other studies, investigators studied how a moving visual environment (Laurens et al., 2010) and/or a sway-referenced platform (Alahmari, Marchetti, Sparto, Furman, \& Whitney, 2014) or the proximity of the visual environment (Bonnet et al., 2010) could influence postural control.

In thi stl dy, our first objective was to analyze the interaction between postural

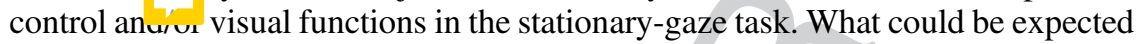
in the relation between eye movement on one hand and center of pressure (COP) and/or body movements (now called COP/body movements) on the other hand in the stationary-gaze task was not obvious. First native correlations could be expecter be ween the eye and COP/body movement if the eyes moved exactly in the opposiu direction and in phase with the COP/body movements. For example, if the body was swaying to the left, the eyes should move to the right with the same amplitude and in phase with the body movements to keep staying on the target. Second $=1$ ly positive correlations between the eye and COP/body movements could be expected it $t$ le correlations between the eye and COP/body movements were only spatial, unat is, not correlated along time. For example, the body could sway to the left and the eyes could move to the right, but with some delays or some imprecisions. Third, there could be no significant correlation between the eye and $\mathrm{COP} /$ body movements, as the eyes move very quickly with no inertia, while the body is heavy. In fact, we expected the second hypothesis to be the best one because there should exist significant relations between the eye and COP/body movements to explain why participants easily succeed in the stationary gaz although unpredictable microsaccades and differences in inertia could impeac perfect subtle relations.

We also studied the relations between the eye and COP/body movements to test-indirectly as ye - the synergistic model of postural control. To clarify this second objective, we irst need to define the synergistic model (Bonnet \& Baudry, 2016) and then explain what the results of the stationary-gaze task could inform us about. In fact, the synergistic model was created to explain/predict relations between the eye and COP/body movements in precise visual tasks, that is, tasks requiring precise gaze shifts on aspects of the visual environment. It was not intended to analyze a stationary gaze in precise visual tasks, our model expects that functional relations should exist between the eye and COP/body movements. This is the most important hypothesis. Here, the term functional relations refers to complementarity between the eye and COP/body movements to succeed in precise gaze shifts. For example, if an individual wants to perform a precise saccade of $10^{\circ}$ on the left, and if he/she sways a corresponding angle of $0.1^{\circ}$ on the left at the same moment, he/she would need to perform a gaze shift of $9.9^{\circ}$ and not $10^{\circ}$ to reach the target straight with no corrective saccade. The model was called synergistic because eye and body movements are expected to be coordinated 
and eo nplementary to succeed in precise visual tasks. However, it should be acknowledged that there is no $m u$ scle or reduction of degrees of liberty in this synergistic model of postural cunol (Bonnet \& Baudry, 2016).

The synergistic model was validated previously in the study by Bonnet, Szaffarczyk, and Baudry (2017). In this study, we found functional relations between the eye and body movements in a search task because healthy young individuals exhibited only negative Pearson correlations between the eye and body (i.e., head and neck) movements. These negative correlations were assumed as functional because the larger the gaze shifts, the lower the individual swayed. In the literature, it is well admitted that a decrease in postural sway is representative of functional, or adaptive, postural control, whereas an increase in postural sway shows a weakened postural control (e.g., Bonnet \& Baudry, 2016; Mitra, Knight, \& Munn, 2013). In the nonprecise control free-viewing task (free-viewing exploration with no goal), we could not find any functional relations, that is, any negative correlations on positive ones. These positive correlations were interpreted as nonfunction... ecause the larger and faster the individuals explored their environment, the larger and faster they swayed, which should be counterproductive to performing precise gaze shifts. In Bonnet et al. (2017), we did not analyze the relations between the eye and COP/body movements in the stationary-gaze task because these relations could not directly test the model. Indeed, there are no precise gaze shifts in this task.

In Bonnet et al. (2017), we also showed that the subjective cognitive involvement was significantly higher in searching than in the free-viewing task Moreover, controlling for the influence of the cognitive involvement canceled alı significant negative correlations between the eye and body movements in the precise search task. We concluded that the central nervous system may have increased its cognitive engagement to better control the upright stance in order to functionally link the eye and body movements in the precise task, whereas it did not do so in the nonprecise visual task (for more details, see Bonnet et al., 2017). One theoretical issue in this study is that individuals performed, on average, longer fixations in searching than in free-viewing task. Long fixations may have had a confounding effect on the results, that is, they may have induced the significant negative vision-posture correlations in searching. Hence, we needed to check whether a fixation task (with long stationary gazes) could actually lead to significant negative correlations between the eye and COP/body movements. If so, the negative correlations found in the search task in Bonnet et al. (2017) would not result from a functional relation between the eye and COP/body movements but would merely depend on longer fixations in the search task $\theta$ rerall, therefore, testing correlations between the eye and COP/body movements in a stationarygaze task could indirectly invalidate the concept of functional relations between the eye and COP/body movements proposed in Bonnet et al. (2017). The term indirect is used here because the synergistic model can only be tested directly with the results of a precise visual task.

The specific analyses of relations between the eye and head movements could also be used to study the vestibulo-ocular reflex (VOR) in a quiet stance; it was a third (secondary) objective of this study. By definition, the VOR is useful to stabilize vision in moving the eyes and head in counterbalanced directions with a ratio close to one in terms of gain (McGarvie et al., 2015; Mossman, Mossman, 
Purdie, \& Schneider, 2015) and almost with no delay (Huterer \& Cullen, 2002;

Sparks, 2002). So far, it does not seem that the eye and head movements were measured simultaneously in healthy young adults to test the functionality of the VOR. Indeed, we could only identify studies testing the VOR in patients (mainly with vestibular problems), older adults, and healthy children. In these studies, the participants performed a quiet stance task and a test in a rotational chair. Then, the amplitude of postural sway measured in the quiet stance was correlated with the gain of the VOR measured in the seated condition (Allum \& Honegger, 2013, 2016; Baloh, Ying, \& Jacobson, 2003; Charpiot, Tringali, Ionescu, Vital-Durand, \& Ferber-Viart, 2010). In this stu ly, we tested if specific correlations between the eye and head movements coulu $u$ positive or negative in an upright stance, and we tested the reflex-like nature of these interactions

In brief, the purpose of this study was to expluic correlations between the eye and COP/body movements specifically in the stationary-gaze task performed upright. A total of 14 healthy young adults performed a main stationary-gaze task. In this task, we rejected the possibility of finding negative significant correlations between the eye and COP/body movements and, instead, expected to find only significant positive ones. The participants also performed a freeviewing task in which they randomly looked at an image. This task was also used to indirectly test the synergistic model. In this free-viewing task, we expected to find only significant positive correlations between the eye and COP/body movements, as in Bonnet et al. (2017). A lower quantity of significant positive correlations and lower correlation coefficients were expected in the free-viewing task than in the stationary-gaze task. In both tasks, we did not expect that changes in cognitive involvement would be required to get the significant correlations between the eye and COP/body movements. Indeed, the stationary gaze may be a reflex-like activity that does not need to engage ad itional cognitive involvement. The free-viewing task should consist of ranc.um.looking gaze shifts that also do not engage any additional cognitive involvement.

\section{Methods}

\section{Participants}

A total of 14 healthy young adults (seven females and seven males) were included in the study. The mean age, body weight, and height were $20.43 \pm 1.70$ years, $68.07 \pm 11.25 \mathrm{~kg}$, and $1.71 \pm 0.1 \mathrm{~m}$, respectively. The study was performed in accordance with the tenets of the Declaration of Helsinki. The participants gave their written, informed consent to participate.

\section{Apparatus}

A dual-top force platform (AMTI, Watertown, MA) was used to record the COP displacement with a sampling frequency of $120 \mathrm{~Hz}$. Three markers of the Polhemus system (LIBERTY 240/8-8 system; Polhemus, Colchester, VT) were placed behind the participants' head, neck, and lower back levels (Figure 1a). They recorded an an zular (yaw, pitch, and roll) and linear (anteroposterior [AP], mediolateral [nL], and vertical) time series with a sampling frequency of 


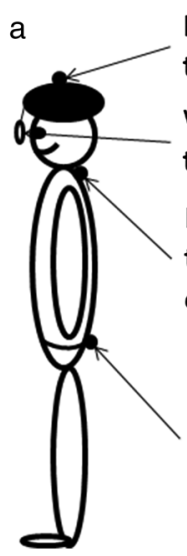

Head marker (on

the helmet)

Window of the eye

tracker

Neck marker (at

the seventh

cervical vertebral

Lower back

marker (on a chest

belt worn by the

b

participants!

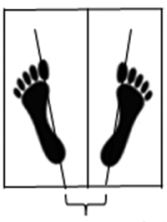

$17 \mathrm{~cm}$ et $14^{\circ} \mathrm{d}^{\prime}$ écart

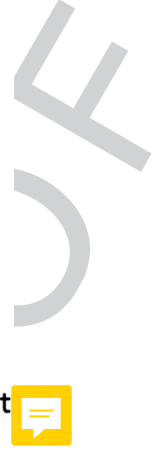

Figure 1 - Two images projected in front of the participants. Only the part within the circle was apparent to the participants (within a circle subtending a visual angle of $22^{\circ}$ ).

$240 \mathrm{~Hz}$. The head and lower back markers were, respectively, placed on a helmet and on a chest belt worn by the participants (Figure 1a). The neck marker was fixed on the neck at the seventh cervical vertebra (Figure 1a) with scotch tape. Ar si II eye tracker (SensoMotoric Instruments, Teltow, Germany) was set on the hemut, and it recorded the position of the eyes with a sampling frequency of $50 \mathrm{~Hz}$. All these devices were synchronized with the images projected $3.40 \mathrm{~m}$ in front of the participants.

A French version (Cegarra \& Morgado, 2009) of the multidimensional National Aeronautics and Space Administration Task Load Index (NASA-TLX) questionnaire (Hart \& Staveland, 1988) was used to record the cognitive workload after each task. As (Bonnet \& Baudry, 2016) 喿 ; questionnaire was chosen because it is a wellvalidated questionnaire sensitive $\ldots$ fine variations between tasks (Cegarra \& Morgado, 2009). This questionnaire already showed significant differences in subjective cognitive engagement between the free-viewing and fixation tasks (Bonnet et al., 2017; Bonnet \& Szaffarczcyk, 2017).

\section{Conditions}

The participants performed three trials in each of the stationary-gaze and freeviewing tasks. In each trial, images of real life in a town (streets, buildings) were projected at eye height onto a circle of $22^{\circ}$ (Figure $2 \mathrm{a}$ and $2 \mathrm{~b}$ ). The three images were the same in both tasks. In the stationary-gaze task, the participants had to fixate on a black cross centered in the circle for the duration of the trial (45 s). In the free-viewing task, the black cross was present for $3 \mathrm{~s}$, and the participants had to look at it. Once the black cross disappeared, the participants could freely look at the image randomly with no goal. In both tasks, they had to avoid any voluntary movement (e.g., hand movements). They were told to relax and hold their arms by the side of the body. The free-viewing task did not serve as a control for the stationary-gaze task, but served to bring complementary data, as discussed later. 

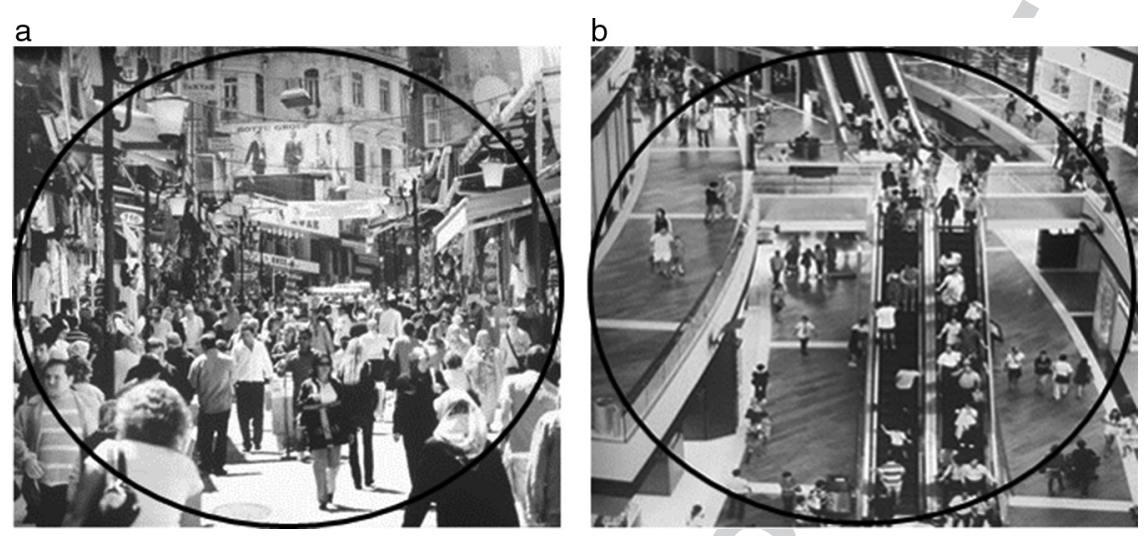

Figure 2 - (a) Image showing the position of the Polhemus markers and of the eye tracker and (b) image showing the position of the feet on the dual-top force platform

\section{Procedure}

During each trial, the participants were barefoot, with their feet positioned along two normative lines (17 cm, 14, cllroy \& Maki, 1997; Figure 1a). The two conditions (st tionary-gaze and $\mathrm{ft}_{1}$ iewing tasks) were run one after another in a counterbainued way. The participants filled the NASA-TLX questionnaire after each task in a seated position (for a rest period of about $5 \mathrm{~min}$ ). Between each trial, the participants could relax for 20-30 s. This duration was required to allow the investigator to record the data and to prepare the next trial.

\section{Variables}

The $S D$, range $(R)$, and mean velocity ( $V$ ) of the COP, markers, and eye displacements were used to analyze the body and eye movements on the AP and ML axes. The path length and ellipse area were also used to provide a general trend of eye and body movements. The ellipse area variable calculated the characteristics of an ellipse, which captured $85 \%$ of the eye movements (Latash, Ferreira, Wieczorek, \& Duarte, 2002). The $S D, R$, and ellipse variables wer $€$ icerned the amplitude of the eye and COP/body movements, whereas the $V$ and paur length were more rel ted to the velocity of the eye and COP/body movements. We computed these twu kinds of movement characteristic to provide a global scheme of relation between the eye and COP/body movements. We expected consistent results with all these variables. The NASA-TLX global score was determined for each task, as recommended (Cegara \& Morgado, 2009; Hart \& Staveland, 1988).

\section{Analyses}

The four analyses performed were as follows: (a) Pearson correlations between the eye and COP/body movements in the stationary-gaze and free-viewing tasks separately; (b) partial correlations between the eye and COP/body movements in 
both tasks separately controlling for-eliminating for-the NASA-TLX's influence on these correlations, and the ;e partial correlations were only performed on the significant Pearson correlauvins found in the previous analyses; (c) crosscorrelation analyses between the eye and COP/body time series in both tasks and both axes separately (ML body movements cross-correlated to left-right eye movements; AP COP/body movements cross-correlated to the corresponding up-down eyes movements); and (d) Pearson correlations between all COP/ body movement variables an all variables of the visual functions te how potential redundant correlations. Thuv per rson correlations were only peiıunned for all the

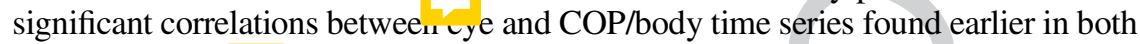
tasks and axes. In Pearson correlations were assumed to look at gross relations between variablu, unly on one value), whereas the cross-correlations were assumed to look at the subtle relations between variables. Indeed, by definition, crosscorrelation is a measure of similarity of (IW) time series as a function of the displacement of one relative to the other (...kipedia). III Pearson correlations searched to know $1 \pm$ ne average quantity of COP/body mo ent could be related to a corresponding average quantity of eye movement. First, the cross-correlations determined the strength of coupling between the eye and COP/body movements for each participant in each task. A first IVI ITAB script was used to resample the COP, head, neck, and lower back time ...es at $50 \mathrm{~Hz}$. A seconc M ATLAB script was used to obtain the cross-correlation coefficients between the eye and COP/body movements for each trial. Second, one-way repeated-measures analyses of variance (factor: tasks) were performed to compare the coefficients in both tasks. Eight analyses of variance were performed between the left/right eye movement and the ML COP, lower back, neck, and head movement, and between the up/down eye movement and the AP COP, lower back, neck, and head movement. All analyses were exploratory and performed with an adjusted $p$ value $(p<.01)$. They were performed with Statistica 10 software (StatSoft Inc., Tulsa, OK). Preanalyzes were performed and only showed an outlier in the data for the ellipse of trunk movement. Hence, this set of data was not analyzed in the correlation analyses later.

\section{Results}

\section{Significant Pearson Correlations Between Eye and COP/Body Movements}

In the stationary gaze, our exploration of significant Pearson correlations between the eye and COP/body movements showed 32 significant positive coefficients (Table 1). Four of these significant correlations are shown in Figure $3 a-3 d$. In the free-viewing condition, no Pearson correlation was significant (Table 1).

\section{Significant Partial Correlations Between Eye and COP/Body Movements, Controlling for the NASA-TLX Global Score's Influence}

In the stationary gaze, three of the 32 significant correlation coefficients with the COP, lower back, neck, and head movements were not significant anymore when 


\section{Table 1 Significant Pearson's Correlations Between Eye and Head, Neck, Lower Back, and COP Movements in the AP and ML Directions and in the Stationary-Gaze and Free-Viewing Tasks $(10<.01)$}

\section{Stationary-gaze task}

Correlations

between eye and head

displacements

Correlations between eye and neck displacements

Correlations between eye and lower back displacements
Correlations between eye and COP displacements
In the up-down/AP directions: $S D$ and $R$ head, $r(16)=.73 ; S D$ and $S D$ head, $r(16)=.75 ; S D$ and ellipse area head, $r(16)=.66 ; p$ th length and $V$ head, $r(16)=.69$; path length and path lengt ad, $r(16)=.70$; ellipse area and Rhead, $r(16)=.72$; ellipse area and $S D$ head, $r(16)=.73$; and ellipse area and ellipse area head, $r(16)=.70$

In the left-right/ML directions: path length and Vhead, $r(16)=.69$, and ellipse area and $R$ head, $r(16)=.66$

In the up-down/AP directions: $S D$ and Rneck, $r(16)=.69 ; S D$ and $S D$ neck, $r(16)=.74 ; S D$ and ellipse area neck, $r(16)=.68$; path length and $V$ neck, $r(16)=.69$; path length and path length neck, $r(16)=.68$; ellipse area and Rneck, $r(16)=.71$; ellipse area and $S D$ neck, $r(16)=.74$; and ellipse area and ellipse area neck, $r(16)=.70$

In the left-right /ML directions: path length and $V$ neck, $\boldsymbol{r}(\mathbf{1 6})=\mathbf{. 6 3}$, and ellipse area and Rneck, $r(16)=.65$

In the up-down/AP directions: $S D$ and Rlower back, $r(16)=.68 ; S D$ and $S D$ lower back, $r(16)=.72 ; S D$ and ellipse area lower back, $r(16)=.67$; path length and Vlower back, $r(16)=.72$; path length and path length lower back, $r(16)=.72$; ellipse area and Rlower back, $r(16)=.73$; ellipse area and $S D$ lower back, $r(16)=.76$; and ellipse area and ellipse area lower back, $r(16)=.71$

In the left-right /ML directions: path length and Vlower back, $r(16)=.63$

In the up-down/AP directions: $S D$ and ellipse area, $r(16)=.63$ In the left-right $/ \mathrm{ML}$ directions: ellipse area and $S D, r(16)=.65$, and ellipse area and ellipse area, $r(16)=.66$

Note. $\mathrm{COP}=$ center of pressure; $\mathrm{AP}=$ anteroposterior; $\mathrm{ML}=$ mediolateral. The above dependent variables of the eyes and COP/body displacements were the range $(R)$, the $S D$ the mean velocity $(V)$, the path length anc ellipse area. The displacements of the eye in the up-d direction were systematically cer 2....... with the displacements of the body on the AP axis, and the displacements of the eyes in the ..... -right direction were systematically con lated with the displacements of the body on the ML axis. However, the variables with no directi ath length or ellipse area) were correlated with the variables in both directions (AP and ML or up-down and left-right). The direction of each variable is only cited at the beginning of each line in Table 1. For each of the 32 significant Pearson correlations, the first variable is systematically the visual variable and the second one is the postural variable. The twe rel tionships in bold were not significant anymore when the influence of the cognitive involve was controlled in partial correlations.

controlling for the NASA-TLX global score's influence. After controlling for this NASA score, (a) the correlation between the $S D$ of the up-down eye movement and the ellipse area of the COP movement changed from $r(16)=.632, p<.01$ (cf. bold result in Table 1) to $r(16)=.631, p=.012$; (b) the correlation between the path length of the eye movement and the mean velocity of the neck movement on 
a

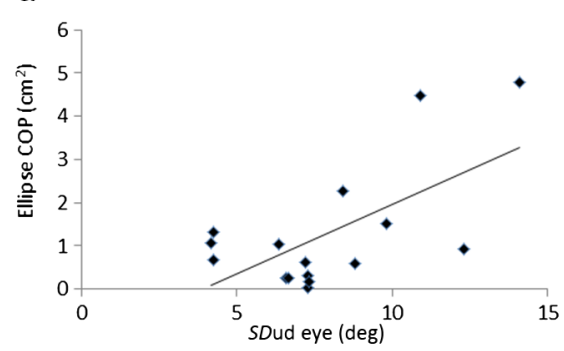

b

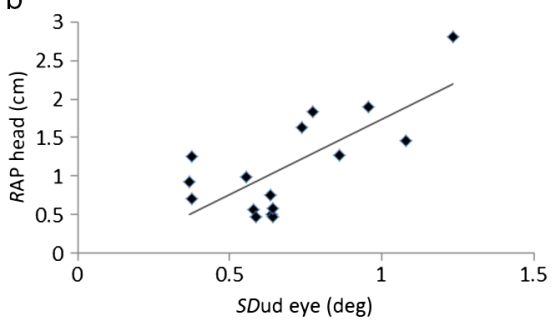

C
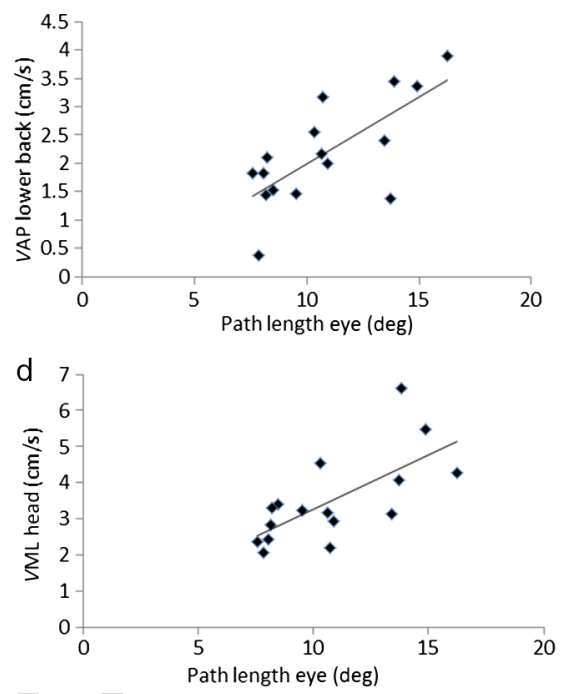

Figure 3 - (a) Significant Pearson correlation $(p<.01)$ between the $S D$ of $\in$ ye movement in the up-down direction (SDud eye, in degrees) and the ellipse area of the center of pressure movement (ellipse COP, in square centimeter), (b) significant Pearson correlation between

Q15 the SDud eye (m legrees) and the range of head movement on the anteroposterior axis (RAP head, in cen......ter), (c) significant Pearson correlation between the path length of eye movement (path length eyes, in degrees) and the velocity of the lower back movement on the anteroposterior axis (VAP lower back, in centimeters per second), and (d) significant Pearson correlation between the path length of the eyes (in degrees) and the velocity of the head movement on the mediolateral axis (VML head, in centimeters per second). $p<.01$.

the ML axis changed from $r(16)=.63, p<.01$ (bold result in Table 1) to $r(16)=.62, p=.013$; and (c) the correlation between the path length of the eye movement and the mean velocity of the lower back movement on the ML axis changed from $r(16)=.63, p<.01$ (bold result in Table 1) to $r(16)=.62, p=.015$.

\section{Significant Difference in Cross-Correlations Between Both Tasks}

The eight cross-correlations (COP, lower back, neck, and head on the AP and ML axes) were not significantly different in the stationary-gaze and free-viewing tasks, $F_{\mathrm{S}}(1,15)<5.32, p>.01$, nonsignifican

\section{Nonredundant Correlations Between Eye and COP/Body Movements}

In Chapter $3.1 \mathrm{w}$ : showed 32 significant correlations between the eye and COP/ body movemenu (Table 1). However, some or many of these correlations could be 
redundant. For this reason, we checked the significant Pearson correlations between all postural variables on one hand and all variables of the visual function on the other hand. These new analyses showed that most of the variables were correlated to one another, and we could only identify four independent correlations between the eye and COP/body movements. These four correlations are shown in Figure $3 \mathrm{a}-3 \mathrm{~d}$. We should note that when many postural and/or visual variables were correlated to the same visual and/or postural variable, we selected the significant correlation with the highest $r$. One should notice that all significant correlation coefficients were close to one another. Hence, the choice of the four specific correlations shown in Figure $3 \mathrm{a}-3 \mathrm{~d}$ merely depends on this criterion; i $1 \mathrm{~s}$ arbitrary anc do not exclude the fact that the relevant correlation could oc different with viner parts of the body movement than the ones described in Table 1.

\section{Discussion}

In this study, we investigated correlations between the eye and COP/body movements in the stationary-gaze and free-viewing tasks performed upright. As expected, the results showed stronger significant correlations in the stationarygaze task than in the free-viewing task; they actually showed significant positive correlations only in the stationary gaze (32 vs. 0). Many of these correlations were redundant, and only four of them were independent of each other. The cognitive involvement was not significantly related to any of the four independent significant correlations and was only significantly related to three of the 32 significant correlations found $(9.4 \%$ ) in is showing that the subjective cognitive involvement may only minimally infl :e them in the stationary gaze. Below, we discuss significant correlations between the eye and head movements in the stationary gaze to explain how these analyses and results could serve to test the functionality of the VOR in the upright stance.

\section{Interest in Visual Functions and Postural Control in the Stationary-Gaze Task}

The visual task of looking at a stationary target upright is an active process (Aytekin \& Rucci, 2012). Indeed, postural sway upright is continuous, irregular, nonlinear, and nonstationary (Bonnet et al., 2010; Collins \& DeLuca, 1995; Riley \& Turvey, 2002), and the eyes are also affected by small unpredictable eye mo vements (Engbert \& Kliegl, 2004; Otero-Millan et al., 2008; Thaler et al., 201\%. Iowever, even in these conditions, individuals perceive a stable visual environment. This visual stability is useful to recognize forms, colors, and textures; to enable the acquisition of visual information (Colagiorgio, Colnaghi, Versino, \& Ramat, 2013); and to avoid visual blurring (Herdman, Schubert, Das, \& Tusa, 2003).

In this study, our main objective was to analyze the linear correlations between the eye and COP/body movements in the stationary-gaze task. We were not aware of any similar literature reports. We found only positive correlations between the eye and COP/body movements in this stationary-gaze task (with the COP, head, neck, and lower back; Table 1, Figure 3a-3d), but no cross-correlation between the eye and COP/body movements. These results, thus, showed that the eye and 
$\mathrm{COP} /$ body movements are not related in a strong manner in a stationary-gaze task performed in a quiet stance. Instead, they were only related in a gross manner (only with mean characteristics of time series), as originally expected. However, it is important to note that the eye and COP/body movements were indeed significantly related to one another in many ways (Table 1).

The results also showed no significant correlation in the free-viewing task (Table 1), thereby vat idating our hypothesis that a stronger link between the eye and COP/body tir... weries could be found in the stationary-gaze task than in the free-viewing task. The strength of the relations in the stationary-gaze task was also higher than the relations in the free-viewing task in our previous study (four positive correlations; Bonnet et al., 2017). ${ }^{1}$ Both umerences in the respective numbers of significant correlations are important because we used the same variables and analyses in both studies. Therefore, the stationary-gaze task firmly required significant relations between the ey an $\mathrm{d}$ body movements, as suggested by Aytekin and Rucci (2012). The number vi significant correlations should be considered cautiously because many of the correlations were redundant, that is, significantly related to each other. Only four correlations between the eye and $\mathrm{COP} /$ body movements were independent of each other (Figure 3a-3d). This redundancy is informative because it showed that similar body movements were performed at different levels of the body, that is, the head, neck, and lower back moved in blocl $\approx$ th respect to the eye movements.

\section{Influence of the Subjective Cognitive Involvement on the Relations Between 티 $\mathrm{e}$ and COP/Body Movements}

In this study, we did not expect to find any significant influence of the cognitive involvement on the correlations between the eye and COP/body movements because both the free-viewing and stationary gaze were easy and basic tasks (Bonnet \& Baudry, 2016). The results indeed validated our hypothesis because in the stationary-gaze task, only $9.4 \%(n=3 / 32)$ of the significant eye-body correlations were influenced by changes in the subjective cognitive involvement, just a little bit above chance (Table $1=$ loreover, none of the four most important relations between the eye and $\mathrm{CC}$., ody movements were significantly related to the cognitive involvement. Hence, the subjective cognitive involvement only marginally changed the correlations between the eye and body movements in the stationary gaze. It should be mentioned that the NASA-TLX questionnaire was useful and appropriately used to study the subjective cognitive involvement in the stationary-gaze task. Indeed, in both Bonnet et al. (2017), and Bonnet and Szaffarczyk (2017), we showed that the NASA-TLX global score was significantly higher in the stationary-gaze task than in the free-viewing task. The stationary-gaze task is indeed psychologically constraining in requires keeping the eyes continuously on a target without looking aro

\section{Indirect Validation of the Cognitive Nature of the Synergistic Model in Both Free-Viewing and Stationary-Gaze Tasks}

The synergistic model expects that there should exist functional relations, that is, negative correlations, between the eye and COP/body movements in precise visual 
tasks, and not in any other visual task. In Bonnet et al. (2017), we indeed hypothesized and to ind only negative correlations between the eye and COP/ body movements in a precise task. We showed that the larger and faster the young participants moved their eyes precisely on the target and the lower and slowed they swayed (cf. Bonnet et al., 2017 . this sti dy had shown significant negative correlations between the eye an $-\mathrm{JP} /$ buuy movements in the stationary gaze and/or in the free-viewing tasks, these results would have caused an issue for the validity of the model. Indeed, these negative correlations in the stationary-gaze task would have suggested that it is not the act of searching that required functional relations between the eye and COP/body movements, as discussed in Bonnet et al. (2017), but simply the act of staring at various targets. In the free-viewing task, if we had found negative correlations between the eye and COP/body movements, it would have suggested that it is not the act of searching that required functional relations between the eye and COP/body movements, but simply the act of moving the eyes randomly on images. Our results, with only positive correlations in the stationary-gaze task (Table 1, Figure $3 a$ and $3 b$ ) and nonsignificant in free-viewing task, thus, indirectly reinforced the validity of the synergistic mode $\underline{m}$ :omplement to Bonnet et al. (2017). Furthermore, the present results did not sho .....ny influence of the subjective cognitive engagement on the four nonredundant (Figure 3a-3d) or on most $(91.6 \%)$ of the 32 significant correlations between the eye and COP/body movements (Table 1) in the stationary-gaze task. We recall that this link definitely existed for the neg ative correlations between the eye and COP/body movements in the precise visuar task in Bonnet et al. (2017; 100\% of the times).

The results of the correlations between the eye and COP/body movements do not have the same meaning in the stationary-gaze versus the free-viewing and search tasks. On one hand, the positive Pearson relations in the slid ionary gaze showed that larger and faster head movements were accompan... by counterbalanced larger and faster eye movements to succeed in keeping the eyes on the stationary target (Table 1). In contrast, the positive Pearson relations in the freeviewing task found in our previous study (Bonnet et al., 2017) most likely showed a destabilizing relation between the eye and COP/body movements because larger and faster eye movements were accompanied by larger and faster COP/body movements to fully explore the image. This situation can be interpreted as destabilizing because gaze shifts cannot be precise when the body moves too much and too quickly. In this situation, lars er eye movements should be accompanied by fewer precision.

\section{Correlations Between Eye and Head Movements in Stationary Gaze}

As stated earlier, the VOR moves the eyes and head in counterbalanced directions with a gain almost equal to one (McGarvie et al., 2015; Mossman et al., 2015) and a very small phase delay of $5 \mathrm{~ms}$ (Huterer \& Cullen, 2002; Sparks, 2002). The literature reports, thus, already showed that eye movements are strongly correlated to head movements. Our results showed 10 significant correlations between eye and head movements (Table 1), thus, validating this general phenomenon. Among these correlations, two were nonredundant significant correlations (Figure $3 \mathrm{~b}$ and $3 \mathrm{~d}$ ). These findings complete the literature reports because they were described 
with correlation analyses and not with a ratio. Moreover, the test was not performed with the participants seated (e.g., Allum \& Honegger, 2013, 2016; Balow er al., 2003; Charpiot et al., 2010), but upright. All the correlation coefficients sho $r$ w in Table 1 were not close to 1 (all $.63<r<.76$ ) as the usual ratio with the VOR gain because the paradigms were different. On one hand, the VOR gain should be high when the body is largely perturbed, for example, when individuals are rotated passively on a rotational chair. On the other hand, the VOR gain should be lower when the body stands quietly upright, as in this stu ly. Accordingly, Aytekin and Rucci (2012) explained that the gain of oculomolur ompensation was lower than 1 when the head was not restrained. The duration of the trials also matters, as it explains this contrast between studies. Both Aytekin and Rucci (2012) and Engbert and Kliegl (2004) explained that the performance of the stationary gaze could not be perfect because of microsaccades. These microsaccades could have influenced our results because the participants looked at the target for a long time (i.e., $45 \mathrm{~s}$ ) and not a few seconds, as in the test with the rotational chair. In conclusion, it seems expected to obtain a high VOR gain and high negative cross-correlations between the eye and head/body movements in dynamic/perturbing situations, but a lower VOR gain and no negative eye and COP/body movements in a quiet stance.

We studied the influence of the subjective cognitive involvement on the 10 significant correlations between the eye and head movements and found no effect at all. Hence, these results validated that our method may capture the reflex nature of these correlations (Table 1). In other words, our analyses and results may test the functionality of the VOR in the upright stance.

\section{Summary and Perspectives}

Altogether, the present exploratory study showed that only significant positive relations between the eye and COP/body movements could be found in the stationary gaze. These results will serve to provide a basis for future studies. This study also showed that these interactions could be reflex-like, as there was almost no influence of the cognitive involvement on these previous results. A limitation of this study relates to the fact that we only used a subjective questionnaire to evaluate the cognitive involvement after each task. A best solution would have been to measure the cognitive involvement during each task (e.g., with measures of electroencephalography or near-infrared spectroscopy) or with the addition of another cognitive task. A future study should take care of this shortcoming and alsc $10 \mathrm{Jk}$ at age-related and disease-related impairments in bott thefficiency to $\ldots u p$ the gaze stationary in a stationary-gaze task. These invesugations are important because postural control seems to decline more rapidly and intensely with age than the VOR gain (Hegeman, Shapkova, Honegger, \& Allum, 2007; McGarvie et al., 2015; Mossman et al., 2015) and because Parkinson disease impairments in eye (Ekker et al., 2017) and motor (Falaki, Huang, Lewis, \& Latash, 2016) relations have already been found.

\section{Note}

1. In Bonnet et al. (2017), we did not check if the four significant correlations could be redundant or not. 


\section{Acknowledgments}

The author would like to thank Sébastien Szaffarczyk (assistant engineer in our laboratory) for his helpful knowledge in programming the synchronization of the various softwares. He also thank: inc Conseil Regional des Hauts-de-France for the visionnAIRR grant (convention $n^{\circ} 160 \quad 3$ ) that supported the work performed by Tanguy Davin (study engineer for this study).

\section{References}

Alahmari, K., Marchetti, G.F., Sparto, P.J., Furman, J.M., \& Whitney, S.L. (2014). Estimating postural control with the balance rehabilitation unit: Measurement consistency, accuracy, validity, and comparison with dynamic posturography. Archives of Physical Medicine and Rehabilitation, 95, 65-73. PubMed ID: 24076084 doi:10.1016/ j.apmr.2013.09.011

Allum, J.H.J., \& Honegger, F. (2013). Relation between head impulse tests, rotating chair tests, and stance and gait posturography after an acute unilateral peripheral vestibular deficit. Otology \& Neurotology, 34, 980-989. PubMed ID: 23820798 doi:10.1097/ MAO.0b013e31829ce5ec

Allum, J.H.J., \& Honegger, F. (2016). Recovery times of stance and gait balance control after an acute unilateral peripheral vestibular deficit. Journal of Vestibular Research, 25, 219-231. PubMed ID: 26890423 doi:10.3233/VES-150561

Aytekin, M., \& Rucci, M. (2012). Motion parallax from microscopic head movements during visual fixation. Vision Research, 70, 7-17. PubMed ID: 22902643 doi:10.1016/ j.visres.2012.07.017

Baloh, R.W., Ying, S.H., \& Jacobson, K.M. (2003). A longitudinal study of gait and balance dysfunction in normal older people. Archives of Neurology, 60, 835-839. doi:10.1001/ archneur.60.6.835

Bonnet, C.T., \& Baudry, S. (2016). A functional synergistic model to explain postural control during precise visual tasks. Gait \& Posture, 50, 120-125. doi:10.1016/j. gaitpost.2016.08.030

Bonnet, C.T., Kinsella-Shaw, J.M., Frank, T.D., Bubela, D., Harrison, S.J., \& Turvey, M.T. (2010). Deterministic and stochastic postural processes: Effects of task, environment, and age. Journal of Motor Behavior, 42(1), 85-97. PubMed ID: 20051351 doi:10. 1080/00222890903498521

Bonnet, C.T., \& Szaffarczyk, S. (2017). The stationary-gaze task should not be systematically used as the control task in postural control. Journal of Motor Behavior, 49, 494-504. PubMed ID: 28033477 doi:10.1080/00222895.2016.1241749

Bonnet, C.T., Szaffarczyk, S., \& Baudry, S. (2017). Functional synergy between postural and visual behaviours when performing a difficult visual task in upright stance. Cognitive Science, 40, 1675-1693. doi:10.1111/cogs.12420

Cegarra, J., \& Morgado, N. (2009, Septembe tude des propriétés de la version francophone du NASA-TLX. In B. Cahour, F eaux, \& A. Giboins (Eds.), EPIQUE 2009: 5ème Colloque de Psychologie Ergonomique (pp. 233-239). Nice, France.

Charpiot, A., Tringali, S., Ionescu, E., Vital-Durand, F., \& Ferber-Viart, C. (2010). Vestibulo-ocular reflex and balance maturation in healthy children aged from six to twelve years. Audiology \& Neurotology, 15, 203-210. PubMed ID: 19893301 doi:10. $1159 / 000255338$

Colagiorgio, P., Colnaghi, S., Versino, M., \& Ramat, S. (2013). A new tool for investigating the functional testing of the VOR. Frontiers in Neurology, 4, 165. doi:10.3389/fneur. 2013.00165 PubMed ID: 24298265 
Collins, J.J., \& De Luca, C.J. (1995). The effects of visual input on open-loop and closedloop postural control mechanisms. Experimental Brain Research, 103, 151-163. PubMed ID: 7615030 doi:10.1007/BF00241972

Einhäuser, W., Moeller, G.U., Schumann, F., Conradt, J., Vockeroth, J., Bartl, K., ... Könic, P. (2009). Eye-head coordination during free exploration in human and cat. Basic and Clinical Aspects of Vertigo and Dizziness, Annals of the New York Academy of Sciences, 1164, 353-366.

Ekker, M.S., Janssen, S., Seppi, K., Poewe, W., de Vries, N.M., Theelen, T., ... Bloem, B.R. (2017). Ocular and visual disorders in Parkinson's disease: Common but frequently overlooked. Parkinsonism and Related Disorders, 40, 1-10. PubMed ID: 28284903 doi:10.1016/j.parkreldis.2017.02.014

Engbert, R., \& Kliegl, R. (2004). Microsaccades keep the eyes' balance during fixation. Psychological Science, 15, 431-436. PubMed ID: 15147499 doi:10.1111/j.0956-7976. 2004.00697.x

Falaki, A., Huang, X., Lewis, M.M., \& Latash, M.L. (2016). Impaired synergic control of posture in Parkinson's patients without postural instability. Gait \& Posture, 44, 209-15 PubMed ID: 27004660 doi:10.1016/j.gaitpost.2015.12.035

Giveans, M.R., Yoshida, K., Bardy, B., Riley, M., \& Stoffregen, T.A. (2011). Postural sway and the amplitude of horizontal eye movements. Ecological Psychology, 23, 247-266. doi:10.1080/10407413.2011.617215

Hart, S.G., \& Staveland, L. (1988). Development of the NASA task load index (TLX): Results of empirical and theoretical research. In P.A. Hancock \& N. Meshkati (Eds.), Human mental workload (pp. 139-183). Amsterdam, The Netherlands: NorthHolland.

Hegeman, J., Shapkova, E.Y., Honegger, F., \& Allum, J.H.J. (2007). Effect of age and height on trunk sway during stance and gait. Journal of Vestibular Research, 17, 75-87. PubMed ID: 18413900

Herdman, S.J., Schubert, M.C., Das, V.E., \& Tusa, R.J. (2003). Recovery of dynamic visual acuity in unilateral vestibular hypofunction, Archives of OtolaryngologyHead \& Neck Surgery, 129, 819-824. PubMed ID: 12925338 doi:10.1001/archotol. 129.8.819

Huterer, M., \& Cullen, K.E. (2002). Vestibuloocular reflex dynamics during high-frequency and high-acceleration rotations of the head on body in rhesus monkey. Journal of Neurophysiology, 88, 12-28. doi:10.1152/jn.2002.88.1.13

Kapteyn, T.S., Bles, W., Njiokiktjien, C.J., Kodde, L., Massen, C.H., \& Mol, J.M. (1983). Standardization in platform stabilometry being a part of posturography. Agressologie, 24, 321-326. PubMed ID: 6638321.

Latash, M.L., Ferreira, S.S., Wieczorek, S.A., \& Duarte, M. (2002). Movement sway: Changes in postural sway during voluntary shifts of the center of pressure. Experimental Brain Research, 150, 314-324. doi:10.1007/s00221-003-1419-3

Laurens, J., Awai, L., Bockisch, C.J., Hegermann, S., van Hedel, H.J.A., Dietz, V., \& Straumann, D. (2010). Visual contribution to postural stability: Interaction between target fixation or tracking and static or dynamic large-field stimulus. Gait \& Posture, 31, 36-41. doi:10.1016/j.gaitpost.2009.08.241

Legrand, A., Mazars, K.D., Lazzareschi, J., Lemoine, C., Olivier, I., Barra, J., \& Bucci, M.P. (2013). Differing effects of prosaccades and antisaccades on postural stability. Experimental Brain Research, 227, 397-405. PubMed ID: 23649967 doi:10.1007/ s00221-013-3519-z

McGarvie, L.A., MacDougall, H.G., Halmagyi, G.M., Burgess, A.M., Weber, K.P., \& Curthoys, I.S. (2015). The video head impulse test (vHIT) of semicircular canal function-Age-dependent normative values of VOR gain in healthy subjects. Frontiers in Neurology, 6, 154. doi:10.3389/fneur.2015.00154 PubMed ID: 26217301. 
McIlroy, W.E., \& Maki, B.E. (1997). Preferred placement of the feet during quiet stance: Development of a standardized foot placement for balance testing. Clinical Biomechanics, 12, 66-70. PubMed ID: 11415674 doi:10.1016/S0268-0033(96)00040-X

Mitra, S., Knight, A., \& Munn, A. (2013). Divergent effects of cognitive load on quiet stance and task-linked postural coordination. Journal of Experimental Psychology: Human Perception and Performance, 39, 323-328. PubMed ID: 23127476.

Mossman, B., Mossman, S., Purdie, G., \& Schneider, E. (2015). Age dependent normal horizontal VOR gain of head impulse test as measured with video-oculography. Journal of Otolaryngology- Head and Neck Surgery, 44, 29. doi:10.1186/s40463015-0081-7 PubMed ID: 30679030.

Otero-Millan, J., Troncoso, X.G., Macknik, S.L., Serrano-Pedraza, I., \& Martinez-Conde, S. (2008). Saccades and microsaccades during visual fixation, exploration, and search: Foundations for a common saccadic generator. Journal of Vision, 8, 1-18. PubMed ID: 19146322 doi:10.1167/8.14.21

Raymakers, J.A., Samson, M.M., \& Verhaar, H.J.J. (2005). The assessment of body sway and the choice of the stability parameter(s). Gait \& Posture, 21, 48-58. PubMed ID: 15536033 doi:10.1016/j.gaitpost.2003.11.006

Riley, M.A., \& Turvey, M.T. (2002). Variability and determinism in motor behavior. Journal of Motor Behavior, 34, 99-125. PubMed ID: 12057885 doi:10.1080/ 00222890209601934

Rougier, P., \& Garin, M. (2007). Performing saccadic eye movements or blinking improves postural control. Motor Control, 11, 213-223. PubMed ID: 17715456 doi:10.1123/ mcj.11.3.213

Schulmann, D.L., Godfrey, B., \& Fisher, A.G. (1987). Effects of eye movements on dynamic equilibrium. Physical Therapy, 67, 1054-1057. PubMed ID: 3602098 doi:10. $1093 / \mathrm{ptj} / 67.7 .1054$

sto fregen, T.A., Bardy, B.G., Bonnet, C.T., Hove, P., \& Oullier, O. (2007). Postural sway and the frequency of horizontal eye movements. Motor Control, 11, 86-102. PubMed ID: 17392569

Thaler, L., Schütz, A.C., Goodale, M.A., \& Gegenfurtner, K.R. (2013). What is the best fixation target? The effect of target shape on stability of fixational eye movements. Vision Research, 76, 31-42. PubMed ID: 23099046 doi:10.1016/j.visres.2012.10.012

Thomas, N.M., Bampouras, T.M., Donova, T., \& Dewhurst, S. (2016). Eye movements affect postural control in young and older females. Frontiers in Aging Neuroscience, 8, 216. PubMed ID: 27695412 doi:10.3389/fnagi.2016.00216 


\section{Queries}

Q1. In the sentence beginning with "As expected, the results showed many $(n=32)$. . ." please consider elaborating upon the phrase "and nonsignificant in free-viewing task."

Q2. In the sentence beginning with "As discussed, these results indirectly strengthened ..." should the opening be "as will be discussed . .."?

Q3. As per journal style, repeats of words in the article title are not allowed in keywords. Hence, the keyword "stationary gaze" was deleted.

Q4. In the sentence beginning with "However, it may not be so easy ..." should "maintain the eyes" be "maintain one's gaze"?

Q5. Please ensure author information is listed correctly here and within the byline.

Q6. Please confirm the use of "impeach" in the sentence beginning with "In fact, we expected the second hypothesis ..." Should this be "impede"?

Q7. Please provide the complete reference details for "Sparks, 2002" to be included in the reference list.

Q8. Please clarify the phrase " $17 \mathrm{~cm}$ et $14^{\circ}$ decart" in Figure 1 .

Q9. The reference citation for "Bonnet et al., 2016" has been changed to "Bonnet \& Baudry, 2016" as per reference list. Please check and verify.

Q10. Please consider rephrasing the sentence beginning with "As (Bonnet \& Baudry, 2016), this questionnaire was chosen . .." for clarity, particularly due to "As (Bonnet \& Baudry, 2016)"

Q11. Please check the terms " $17 \mathrm{~cm}, 14$ " in the sentence "During each trial ..." for clarity.

Q12. Please provide the complete reference details for "Cegara \& Morgado, 2009 " to be included in the reference list.

Q13. In the sentence containing "Pearson correlations searched to know if ..." Please consider rewording "searched to know if" You might consider "investigated whether"

Q14. In Table 1, please note that "Free-Viewing Tasks" is specified in table caption but not provided in the table. Please check.

Q15. In the section that contains "(b) significant Pearson correlation between the SDud eye ..." Please confirm SDud

Q16. In the sentence "The eight cross-correlations ...," ns has been spelled out as nonsignificant. Please check.

Q17. Please specify the appropriate section/subsection head instead of mentioning "Chapter 3.1."

Q18. Please check the sentence "Hence, the choice ..." for clarity.

Q19. Please confirm the suggested change from "eyes" to "eye" in the sentence 
starting with "Therefore, the stationary-gaze task firmly required significant ...."

Q20. Please confirm the use of "moved in block" in the sentence beginning with "This redundancy is informative because it showed . . ."

Q21. Please confirm the suggested punctuation change in the sentence beginning with "The results indeed validated our hypothesis because in the . . .."

Q22. Please consider rephrasing the sentence beginning with "We showed that the larger and faster the young participants moved their eyes . . ." for clarity.

Q23. Please consider adjusting "in complement to" in the sentence beginning with "Our results, with only positive correlations ....."

Q24. Please provide the complete reference details for "Balow et al., 2003" to be included in the reference list.

Q25. Please check the sentence "A future study ..." for clarity.

Q26. Please consider adjusting the sentence beginning with "A future study should take care of this shortcoming . . ." due to the placement of "both."

Q27. Please check and correct the phrase "Conseil Regional des Hauts-deFrance for the visionnAIRR grant (convention $n^{\circ} 16000913$ )" in the sentence "He also thank . . .."

Q28. Please provide the English translation for chapter and book titles, and publisher location for reference "Cegarra and Morgado (2009)."

Q29. Please provide in-text citation for the reference "Einhäuser et al., 2009." 